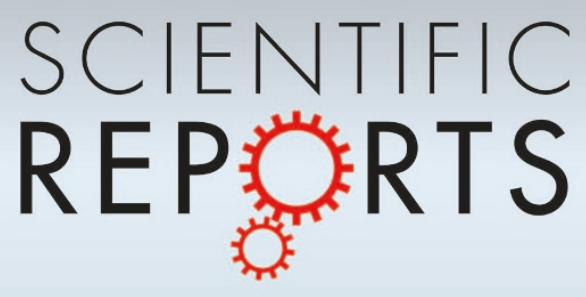

\title{
open Quorum quenching bacteria Bacillus sp. \\ BACTERIAL QSI-1 protect zebrafish (Danio rerio) from Aeromonas hydrophila infection
}

SUBJECT AREAS:

PATHOGENESIS

MICROBIOLOGY TECHNIQUES

Received

21 January 2014

Accepted

9 June 2014

Published

25 June 2014

Correspondence and requests for materials should be addressed to

W.C. (chuweihua@ cpu.edu.cn)

Weihua Chu', Shuxin Zhou'', Wei Zhu' \& Xiyi Zhuang'

\author{
'Department of Pharmaceutical Microbiology, China Pharmaceutical University, Nanjing 210009, P. R. China, ${ }^{2}$ School of \\ International Pharmaceutical Business, China Pharmaceutical University, Nanjing 210009 , P. R. China.
}

Quorum Sensing (QS) is a bacterial regulatory mechanism, which is responsible for controlling the expression of various biological macromolecules such as the virulence factors in a cell density-dependent manner. Disruption of the QS system of pathogens has been proposed as a new anti-infective strategy. Biodegradation of AHLs proves to be an efficient way to interrupt QS, since AHLs are the main family of QS autoinducers used in Gram negative bacteria. In this study, the effect of Bacillus sp. QSI-1 as an efficient quorum quencher on virulence factors production and biofilm formation of fish pathogen Aeromonas hydrophila was investigated. QSI-1 reduced the accumulation of AHLs but did not affect the growth of $A$. hydrophila YJ-1 when cocultured. In the result, the supernatant of QSI-1 showed significant inhibition of protease production (83.9\%), hemolytic activity $(77.6 \%)$ and biofilm formation $(77.3 \%)$ in YJ-1. In biocontrol experiment, QSI-1 significantly reduced the pathogenicity of $A$. hydrophila strain YJ-1 in zebrafish (Danio rerio). The fish fed with QSI-1 was observed to have a relative percentage survival of $80.8 \%$. Our results indicate that AHLs degrading bacteria should be considered as an alternative for antibiotics in aquaculture for the biocontrol of bacterial fish diseases.

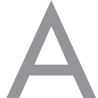

ntibiotics are critical in preventing, controlling, and treating disease in farm animals. By preventing essential processes such as cell wall synthesis, DNA proliferation, and protein synthesis, antibiotics become an integral part in keeping our food supplies safe from infectious bacteria. Unfortunately, the widespread use of antibiotics eventually lead to the selection of bacteria which is resistant to the antibiotic. These antibiotic-resistant bacteria population render the antibiotics ineffective, and new antibiotics are needed ${ }^{1}$. In order to combat pathogenic bacteria without fear of developing an antibiotic resistant strain, alternative methods need to be investigated.

Quorum sensing (QS) is a mechanism in which bacteria coordinates gene expressions in response to their population density by producing, releasing, and recognizing small signal molecules called autoinducers ${ }^{2,3}$. QS regulates various phenotypes such as biofilm formation ${ }^{4-7}$, bioluminescence ${ }^{8-11}$, virulence factors ${ }^{12}$ and swarming $^{13,14}$ which have been shown it's contribute to bacterial pathogenesis. Since pathogenicity traits of bacteria are controlled by QS, disruption of quorum sensing QS has been suggested as a new anti-infective strategy to control pathogenic bacteria, without interfering with their growth ${ }^{15,16}$, in the field of aquaculture and animal husbandry ${ }^{17,18}$. Quorum quenching (QQ), the disruption of QS, can be performed by small molecule antagonists ${ }^{19}$ or signal degrading enzymes ${ }^{20}$. Many microorganisms can produce enzymes which can degrade N-Acylhomoserine lactones (AHLs) ${ }^{21-23}$. The QQ enzymes produced by microorganisms were classified into three major types according to their enzymatic mechanisms: AHL lactonase (lactone hydrolysis), AHL acylase (amidohydrolysis) and AHL oxidase and reductase (oxidoreduction). These enzymes can degrade AHLs, and as a consequence, prevent pathogenic bacteria from producing virulence factors, forming biofilms, and reducing virulence. So the $\mathrm{QQ}$ microorganisms can be used as potential quenchers of quorum-sensing-regulated functions in pathogenic bacteria ${ }^{24}$. Therefore it could act as an alternative to antibiotics.

In the present study, we investigate the previously isolated bacterium with quorum quenching activity, Bacillus sp. QSI- ${ }^{25}$, at degrading AHL signals of A. hydrophila and interrupting of its QS functions. Furthermore, we investigate the effect of QSI-1 quorum quenching activity on the pathogenicity of $A$. hydrophila in zebrafish. The results demonstrate its potential as an environmentally friendly alternative to antibiotics in aquaculture. 
$\mathrm{Oh}$

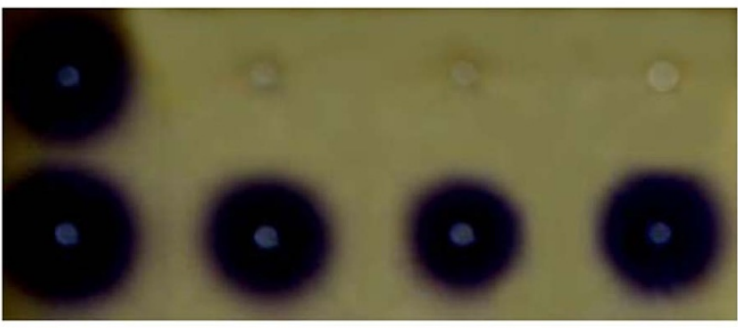

Figure $1 \mid$ Degradation of C6-HSL by QSI-1. Disappearance of C6-HSL was revealed by decreased or loss of purple pigmentation on the biosensor C. violaceumCV026 lawn. From left to right, degradation of C6-HSL cocultured with Bacillus sp. QSI-1 at 0, 6, 12, and $24 \mathrm{~h}$.

\section{Results}

AHL inactivation assay. Inactivation of C6-HSL was confirmed with the loss of purple pigmentation using the resting cells of QSI-1 at different time intervals at $(0,6,12,24$ hours) and at different concentrations of $(4 \mu \mathrm{l}, 6 \mu \mathrm{l}, 8 \mu \mathrm{l}, 10 \mu \mathrm{l})$ by using $C$. violaceum CV026 as bio-reporter strain. Our results suggested that QSI-1 showed high AHL degrading ability even at $0.1 \mu \mathrm{g} / \mu \mathrm{l}$ C6-HSL within $6 \mathrm{hrs}$ and no AHL molecules were found after further incubation to $24 \mathrm{~h}$ (Fig. 1). Disappearance of purple pigmentation production was also observed with the quorum quenching activities of QSI-1 supernatant on CV026 lawn. Nearly all C6-HSL was degraded after 8 hours of incubation. Our results showed that QSI-1 can degrade AHLs and at the same time, the metabolites of QSI-1 also can degrade AHL.

Bacillus sp. QSI-1 blocked AHL signal in A. hydrophila accumulation but did not affect its growth. From antagonistic assay, the Bacillus sp. QSI-1 did not have any negative effect on YJ-1, since YJ-1 grew properly in presence of QSI-1 filtered supernatant (Fig. 2). To test the effect of Bacillus sp. QSI-1 on AHL accumulation and growth of A. hydrophila YJ-1, YJ-1 was cocultured with or without Bacillus sp. strain QSI-1. AHL autoinducer was evaluated on LB agar, again using CV026 as a biosensor. In single culture, AHL produced by strain $\mathrm{YJ}-1$ was detectable $3 \mathrm{~h}$ after inoculation, and a rapid increase was observed between 8 to $10 \mathrm{~h}$ after incubation, parallelling to the exponential proliferation stage of the bacterial cells (Table 1). However, no AHL was detected in the culture supernatant of YJ-1 co-cultured with QSI-1, which produce AHLlactonase. In the competition assay, when A. hydrophila was grown with or without Bacillus sp. QSI-1, the obtained results showed no considerable variation in values of bacterial growth curve of both QSI-1-treated and untreated bacterial cultures of A. hydrophila YJ1. This indicates that, Bacillus sp. had no effect on the growth of YJ-1 (Fig. 3).

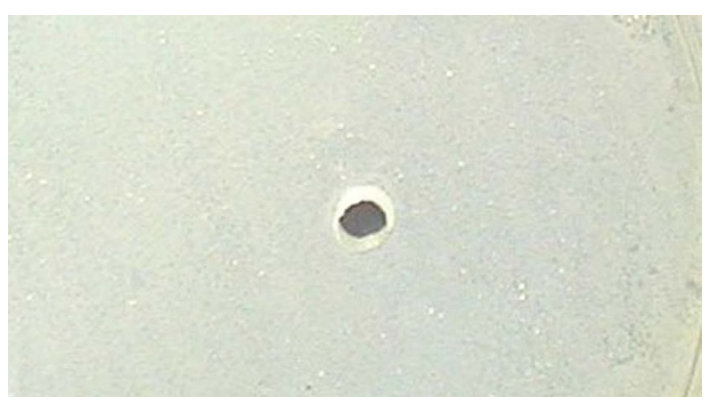

Figure 2 Antagonistic assay of filtered supernatant of Bacillus sp. QSI-1 on A. hydrophila YJ-1.

\begin{tabular}{|c|c|c|c|c|c|c|c|}
\hline \multirow{2}{*}{$\frac{\text { Growth time }}{\text { Supernatant }}$} & \multicolumn{7}{|c|}{ Purple pigment zone formation in $\mathrm{mm}$} \\
\hline & $\mathrm{Oh}$ & $3 \mathrm{~h}$ & $5 \mathrm{~h}$ & $8 \mathrm{~h}$ & $10 \mathrm{~h}$ & $24 \mathrm{~h}$ & $36 \mathrm{~h}$ \\
\hline YJ-1 & - & 6 & 7 & 8 & 11 & 15 & 15 \\
\hline YJ-1-QSI-1 & - & - & - & - & - & - & - \\
\hline
\end{tabular}

QSI-1 supernatant suppressed the virulence factors production and biofilm formation of $\boldsymbol{A}$. hydrophila. The results of AHL inactivation indicated that Bacillus sp. QSI-1 can inhibit QS in $C$. violaceum, and therefore the supernatant of QSI-1 was further assay with YJ-1. YJ-1 is known for its ability to secrete several hydrolytic enzymes including toxins and proteases into the environment, which is associated with the virulence of this opportunistic pathogen. The expression of these virulence factors is under the control of QS system. Thus, in this present work, the impact of QSI-1 supernatant in inhibiting the QS-dependent proteolytic and hemolytic activity of $A$. hydrophila YJ-1 was determined. The total protease activity in supernatants from $A$. hydrophila YJ-1 grown without QSI supernatant was $0.756 \Delta \mathrm{A} 400 \mathrm{~nm}$ after $24 \mathrm{~h}$ whereas the presence of QSI supernatant resulted in a significant decrease in protease activity which was $0.122 \Delta \mathrm{A} 400 \mathrm{~nm}$ (Fig. $5 \mathrm{~A}$ ). To determine whether supernatants of the Bacillus sp. QSI-1 affected the $\beta$-hemolytic activity of YJ-1, sheep blood agar plates were used to roughly detect. The blood agar plate added with the mixture of YJ-1 and QSI-1 supernatant showed small hemolytic zone then YJ-1 supernatant (Fig. 4). Hemolytic activity in liquid assay indicated that YJ-1 produce $77.6 \%$ less hemolysin when adding QSI-1 supernatant (Fig. 5 B). A. hydrophila has the ability to form biofilm, a partially QS dependent phenomenon ${ }^{26}$. Hence, an investigation was carried out to study antibiofilm ability of Bacillus sp. QSI-1 supernatant against YJ-1. After $24 \mathrm{~h}$ culture $200 \mu \mathrm{g} / \mathrm{ml}$ of QSI-1 supernatant led to $77.3 \%$ reduction in biofilm formation (Fig. 5 C).

Effect of QSI-1 A. hydrophila YJ-1 infection. The virulence of A. hydrophila strain YJ-1 to zebrafish was determined. The $\mathrm{LD}_{50}$ of $A$. hydrophila YJ-1 was $1.8 \times 10^{5} \mathrm{CFU}$ to zebrafish. Most dying fish showed clinical signs typical of hemorrhagic septicemia. Colonies of the A. hydrophila strain was recovered from infected fish, and no evident external injuries were observed in the surviving fish. According to the degree of virulence described by Mittal et al. ${ }^{27}$, The pathogenicity of strain YJ-1 to zebrafish was included in the virulent category (LD50s of $10^{2} \sim 10^{5}$ ).

In the challenge test, high survival was observed in QSI-1 fed group on 2 weeks after challenge. It was found that the survival rate of QSI-1 diet group was $83.3 \%$ (25/30) and the survival rate of PBS control group was only $13.3 \%$ (4/30). After 2 weeks of challenge, the relative percentage survival of QSI-1 fed fish group was raised up to $80.8 \%$. Some of the diseased fish in the experimental group as well as the control groups developed the typical symptoms of $A$. hydrophila infection on the external body surface and showed severe skin lesions such as prominent hemorrhages. However, the infected area of the body surface was smaller in QSI-1 fed fish compared with control group and slowly disappeared after continuously fed with QSI-1. Atypical A. hydrophila was isolated from hemorrhagic lesions of both dead and survived fish in control groups (Fig. 6).

\section{Discussion}

It has been demonstrated that the QQ enzymes could reduce the virulence of pathogenic bacteria. Zhang, et. al., expressed the aiiOAIO6 in Escherichia coli. They also studied the effects of purified enzyme solution on the expression of virulence factors. Their results showed that AiiO-AIO6 could inhibit A. hydrophila virulence factors 


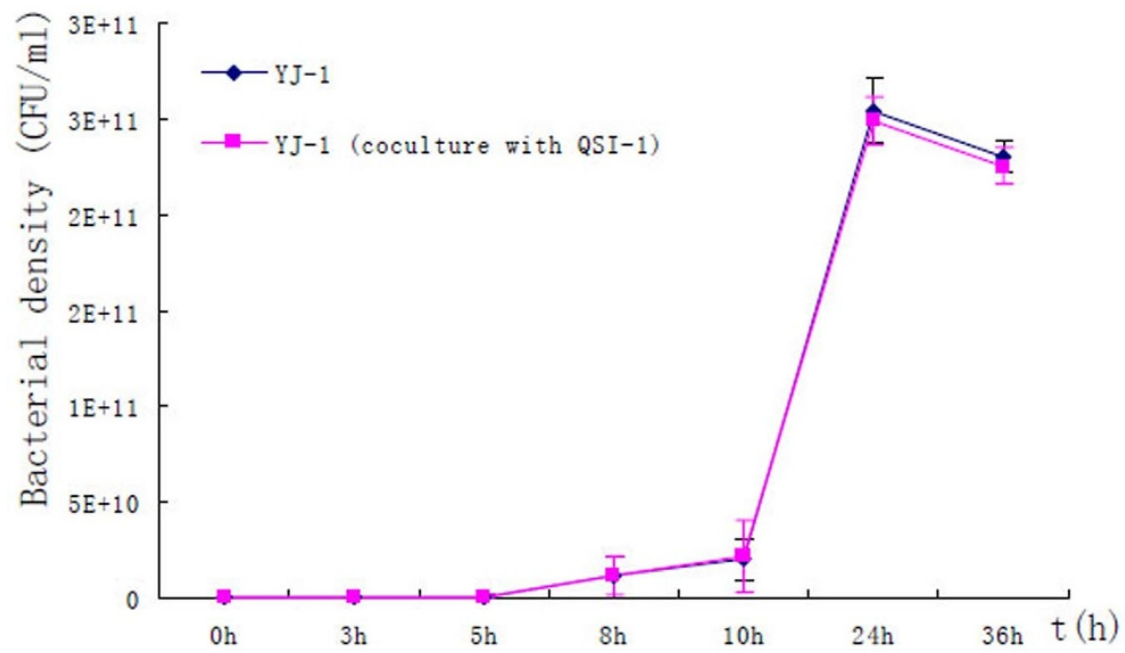

Figure 3 Influence of Bacillus sp. QSI-1 on the growth of A. hydrophila YJ-1.

expression $^{28}$. Cao et. al., supplied the expressed AiiAAI96 into fish feed and found that the oral administration of AiiAAI96 significantly decreased the mortality of infected zebrafish ${ }^{29}$. In the present investigation, we showed the quorum quenching strain Bacillus sp. QSI-1 which originally isolated from the gut of health Carassius auratus gibelio, can inhibit the AHL-dependent violacein production in $C$. violaceum and virulence factors such as protease, hemolysins production and biofilm formation in A. hydrophila YJ-1. When QSI-1 cocultured with YJ-1, there is no effect on the growth of YJ-1, but the production of quorum sensing molecules was decreased compared with the mono-culture of YJ-1. Similar to Zhang et. al.'s results, the total proteolytic, hemolytic activity and biofilm formation of YJ-1 were significantly decreased when added with the supernatant of QSI-1. In our protection ability experiments, QSI-1 was supplemented into experimental diets to control $A$. hydrophila infection. The results showed that the oral administration of QSI-1 significantly decreased the mortality of infected zebrafish. This result may provide a new explanation of modes of action of probiotics.

In summary, in this study we demonstrated yet another pharmaceutically important property of bacterial resources against bacterial QS systems and a new way to combat bacterial diseases instead of antibiotics in aquaculture. The results of the present study clearly show a significant decrease in the QS-mediated phenotypic expression of the target bacterial pathogens without any reduction in its growth. And fish experiments also showed that the QQ bacteria can increase the survival rate. Owing to the importance of QS during bacterial pathogenesis, isolated compounds from Bacillus sp. QSI-1 will be a promising tool to human health as they prevent the onset of bacterial diseases as anti-pathogenic drugs, which is not subject to antibiotic resistance.

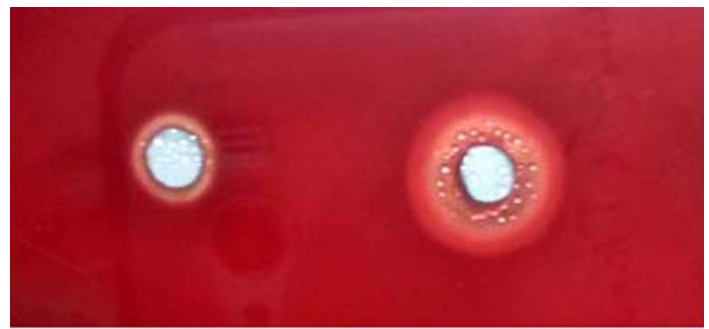

$$
\text { YJ-1 + QSI-1 supernatant YJ-1 supernatant }
$$

Figure $4 \mid$ Hemolysis activity on sheep erythrocytes LBA plate.

\section{Methods}

Bacterial strains and culture conditions. The quorum quenching strain Bacillus sp. QSI-1 which was isolated from the intestine gut of health Carassius auratus gibelio was cultivated in Luria-Bertani (LB) broth consisting of $1 \%$ tyrptone, $0.5 \%$ yeast extract and $0.5 \% \mathrm{NaCl}(\mathrm{pH} 7.4 \pm 0.2)$, and incubated for $24 \mathrm{~h}$ at $30^{\circ} \mathrm{C}$. The cell-free supernatant from QSI-1 was obtained by centrifugation at $12,000 \mathrm{rpm}$, the supernatant filtered through $0.22 \mu \mathrm{m}$ membrane filter. The filtered supernatant was then extracted with an equal volume of ethyl acetate; the solvent layer was separated and evaporated to dryness. The dried extract was weighed and used for anti-QS bioassays. The target pathogen A. hydrophila YJ-1 used in this study was isolated from a diseased fish. It was cultivated in Luria-Bertani (LB) broth $(\mathrm{pH} 7.2 \pm 0.2)$ at $150 \mathrm{rpm}$ in a shaker overnight at $28^{\circ} \mathrm{C}$. CV026, a mini-Tn5 mutant derived from a Chromobacterium violaceum strain ${ }^{30}$ was used as an AHL-reporter to detect the residual AHL concentration in the rearing water. This mutant cannot produce AHL, but it can detect and respond to a range of AHL molecules (with acyl chain of four to eight carbons), by synthesis of the purple pigment violacein. CV026 was grown in LB at $30^{\circ} \mathrm{C}$. This medium was supplemented with $20 \mathrm{mg} \mathrm{l}^{-1}$ of kanamycin.

AHL inactivation assay. AHL inactivation assays was conducted as described by Chan et. $\mathrm{al}^{21}$. QSI-1 was grown overnight at $30^{\circ} \mathrm{C}$ with shaking $(220 \mathrm{rpm})$ in LB medium to approximately $10^{9} \mathrm{cfu} / \mathrm{ml}$, cells $(100 \mathrm{ml})$ were collected by centrifugation, washed and resuspended in $10 \mathrm{ml}$ of PBS $(100 \mathrm{mM}, \mathrm{pH}$ 6.5) equalized to an OD of 1.0 at $600 \mathrm{~nm}$ and used directly as a source of resting cells for in vitro AHL inactivation assays. Aliquots of C6-HSL $(10 \mu \mathrm{g} / \mu \mathrm{l})$ (Sigma-Aldrich, St. Louis, Missouri, USA) in absolute ethanol were dispensed into sterile tubes and the solvent evaporated to dryness under sterile condition. The resting cells were used to rehydrate the C6-HSL to a final concentration of $0.1 \mu \mathrm{g} / \mu \mathrm{l}$. The mixtures were incubated at $37^{\circ} \mathrm{C}$ for $4.5 \mathrm{~h}$ with gentle shaking in a hybridization oven. Heat-denatured suspension $(10 \mu \mathrm{l})$ was inoculated onto LB agar seeded with the bio-reporter CV026 and incubated at $28^{\circ} \mathrm{C}$. E. coli strain DH5 $\alpha$ served as negative controls. Disappearance of C6-HSL from the mixture was assessed at different time using CV026 bioreporter. Degradation of C6HSL is evident by loss of purple pigmentation shown by C. violaceum and the results were digitally recorded.

For AHLs activity in the supernatant produced by A. hydrophila, the well-diffusion assay was used. Ten milliliters of warm molten soft top LB agar ( $0.6 \%$ agar) was seeded with $100 \mu \mathrm{L}$ of an overnight CV026 culture. This was gently mixed and poured immediately over the surface of a solidified LBA plate as an overlay. Wells of $5 \mathrm{~mm}$ in diameter were made on each plate after the overlay had solidified. Each well was filled with $50 \mu \mathrm{L}$ of filter sterilized culture extract.

The plates were incubated for $48 \mathrm{~h}$ at $28^{\circ} \mathrm{C}$ and the diameter of pigment production by the monitor strain measured.

In vitro competition assay between Bacillus sp. QSI-1 and A. hydrophila. This experiment was done in two different steps; First the antagonistic activity of Bacillus sp. QSI-1 against $A$. hydrophila YJ-1 was evaluated. LB broth cultures were prepared freshly from the A. hydrophila agar slopes (stored at $4^{\circ} \mathrm{C}$ ) and were incubated on $\mathrm{LB}$ agar plates (diameter, $98 \mathrm{~mm}$ ) separately using a lawn culture technique ${ }^{31}$. Then, a 6$\mathrm{mm}$-diameter well was made in each plate with the help of a cork borer. A 100- $\mu \mathrm{l}$ aliquot of cell-free supernatant was charged in the well and incubated at $37^{\circ} \mathrm{C}$ for $24 \mathrm{~h}$. The zones of inhibition around the well was recorded (in millimetres) after the incubation. Control plates were simultaneously maintained with sterile phosphate buffer saline ( $\mathrm{pH} 7.2)$ poured into respective well prepared as mentioned above. Triplicate plates were maintained along with the PBS control for the antagonistic activity study.

In the second step in order to investigate the effect of QSI-1 on the growth of $A$. hydrophila YJ-1was evaluated in mixed liquid cultures. Erlenmeyer flasks containing 
$100 \mathrm{ml}$ of LB were inoculated with $100 \mu \mathrm{l}$ of independent overnight cultures of the Bacillus sp. strain QSI-1 and A. hydrophila YJ-1. The turbidity of the overnight cultures was measured at $600 \mathrm{~nm}$ and adjusted with sterile LB medium to obtain inoculums with the same cell ratio. The inoculated flasks were incubated at $30^{\circ} \mathrm{C}$ with shaking at 150 rpms in an orbital incubator. Samples were collected after $0,3,5,8,10$ and 24 hours. For cells detection, $500 \mu \mathrm{l}$ of culture were removed and after proper dilutions in PBS, all cultures were plated on LB agar plates. After 16-18 h cultured at $30^{\circ} \mathrm{C}$, A. hydrophila and Bacillus sp. strain QSI-1 colonies were easily distinguishable based on their unique colony morphologies (Fig. 7). The experiment was repeated four times.

Effect of supernatant of QSI-1 on virulence factors production and biofilm formation in A. hydrophila. A. hydrophila YJ-1 cultures was grown for $24 \mathrm{hrs}$ to
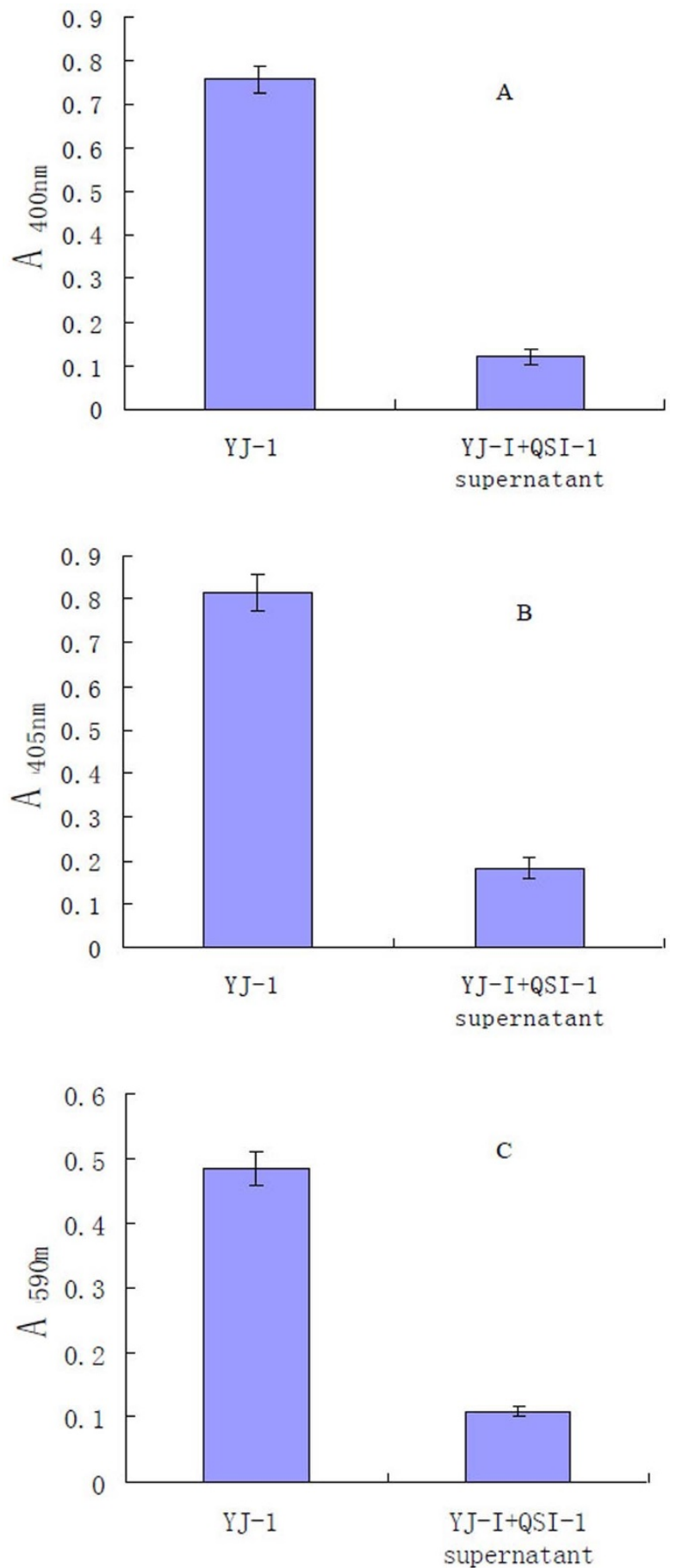

Figure $5 \mid$ Effect of Bacillus sp. QSI-1 supernatant on AHL-mediated factors in A. hydrophila YJ-1. (A) Total protease, (B) Hemolytic activities, and $(\mathrm{C})$ Biofilm formation. The data represent the mean values of three independent experiments and are presented as mean \pm SD.
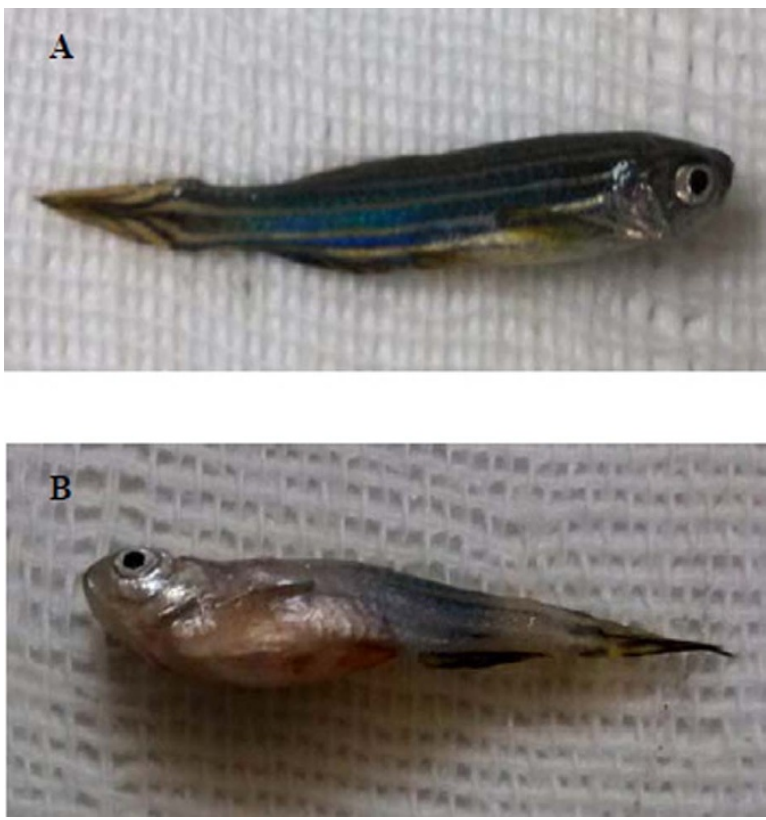

Figure $6 \mid$ Zebrafish with good body condition (A) and infected with Ah YJ-1 (B).

O.D600 of 2.0 (for the protease, hemolytic activity and biofilm formation assays) treated with $200 \mu \mathrm{g} / \mathrm{ml}(\mathrm{w} / \mathrm{v})$, Bacillus sp. QSI-1 supernatant in $2 \mathrm{ml}$ fresh LB medium. Cells were harvested by centrifugation and the supernatant were filter sterilized. The supernatant were either used immediately or stored at $-20^{\circ} \mathrm{C}$.

Proteolytic activity was determined by azocasein assay by following the method of Swift et al. ${ }^{32}$ with slight modifications. Briefly, $150 \mu \mathrm{l}$ of both treated and untreated YJ-1 culture supernatants was added to $1 \mathrm{ml}$ of $0.3 \%$ azocasein (Sigma, St. Louis, USA) in $0.05 \mathrm{M}$ Tris- $\mathrm{HC} 1$ and $0.5 \mathrm{mM} \mathrm{CaCl} 2\left(\mathrm{pH} 7.5\right.$ ), and incubated at $37^{\circ} \mathrm{C}$ for $15 \mathrm{~min}$ respectively. The reaction was stopped by the addition of trichloroacetic acid $(10 \%, 0.5 \mathrm{ml})$ followed by centrifugation, and the absorbance of clear supernatant was measured at $400 \mathrm{~nm}$ in a UV-visible spectrophotometer.

Blood agar, for detecting secreted hemolytic activity, was prepared by adding $5 \%$ washed erythrocytes to LBA ${ }^{33}$. Hemolysis activity in liquid assay was determined as described previously ${ }^{34}$. Briefly, serial dilutions of hemolytic samples were prepared with phosphate-buffered saline (PBS). $100 \mathrm{ml}$ aliquots of each dilution were incubated with $1 \mathrm{ml}$ of erythrocyte suspension for $15 \mathrm{~min}$ at $25^{\circ} \mathrm{C}$ and then centrifuged for $1 \mathrm{~min}$. The fluorescence absorbance at $405 \mathrm{~nm}$ (A405) of released hemoglobin was measured. Hemolytic activities are presented as the percentage of the total erythrocytes lysed (percent hemolysis). The percent hemolysisis defined as [ $\left(\mathrm{A}_{405}\right.$ for the

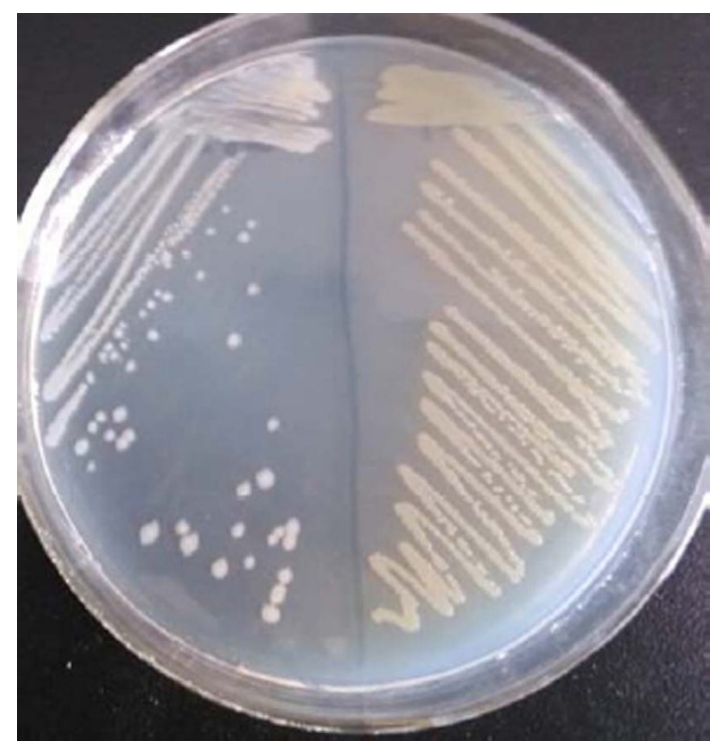

Figure $7 \mid$ Colonies of Bacillus sp. QSI-1 (left) and A. hydrophila YJ-1 (right) on LB agar plate. 
sample with hemolysin $-\mathrm{A}_{405}$ for the control without hemolysin) $\left.\times 100\right] /(\mathrm{A} 405$ for total lysis caused by sodium dodecylsulfate - A405 for the control).

Standard Tube Method (STM) was used for quantitative analysis of biofilm production ${ }^{35}$.Briefly, overnight bacterial cultures were inoculated into $5 \mathrm{ml} \mathrm{LB}$ medium with $1: 1000$ dilution and incubated in the borosilicate glass tubes without shaking at $28^{\circ} \mathrm{C}$ for $48 \mathrm{~h}$. After the bacterial cultures were poured out, the tubes were washed extensively with water, then fixed with $2.5 \%$ glutaraldehyde, followed by washing once with water, and staining with a $0.4 \%$ crystal violet solution. After solubilization of the crystal violet with ethanol-acetone $(80: 20, \mathrm{vol} / \mathrm{vol})$, the absorbance at $590 \mathrm{~nm}$ was determined. The experiment was performed in triplicate and repeated three times.

Fish experiment. Wild-type zebrafish were brought from an ornamental fish farm in Nanjing, Jiangsu Province, and are allowed to acclimate for at least 3 days before use. Basal diet was prepared as mentioned elsewhere ${ }^{36}$. For $50 \%$ lethal dose (LD50) determinations, seven groups of 10 Wild-type zebrafish (4 months of age, with an average weight of $\sim 300 \mathrm{mg}$ and an average length of $\sim 2.5 \mathrm{~cm}$ ) intraperitoneally injected with $0.02 \mathrm{ml}$ of serially tenfold diluted bacterial suspensions containing 101108 CFU. A control group was injected intraperitoneally with $0.02 \mathrm{ml}$ sterile PBS only. The fish were monitored at $28^{\circ} \mathrm{C}$.for mortality for 7 days. During this period, activity and behavior of fish were recorded daily, and LD50 were calculated by the method of Reed and Muench ${ }^{37}$

For QSI-1 contained diet, the QSI-1 was cultured for $24 \mathrm{~h}$ at room temperature (27 $\pm 2^{\circ} \mathrm{C}$ ) in $\mathrm{LB}$ medium, and cells were harvested by centrifugation at $10,000 \mathrm{rpm} 4^{\circ} \mathrm{C}$ for $20 \mathrm{~min}$. The pellets were resuspended in PBS at the concentration of $10^{10} \mathrm{cfu} / \mathrm{ml}$ and mixed with feed to contain the final count of $10^{8} \mathrm{cfu} / \mathrm{g}$ of feed and sun dried. Prior to the experiment, the amount of diet consumed per fish was monitored for 7 days, and based on the data, the QSI-1 amount in the experimental feeds was adjusted approximately $4.0 \times 10^{8}$ cells per $\mathrm{g}$ fish day. Control fish were fed with the dry pellet without QSI-1 bacterial culture.

Before challenge, all fish were clinically healthy and there was no evidence of $A$. hydrophila infection, nor was there any death due to $A$. hydrophila infection. Control and QSI-1 consumed fish (30 per group) were challenged via intramuscular injection with $0.1 \mathrm{ml} \mathrm{PBS}$ or $6.0 \times 106$ cells $(5 \times$ LD50) of A. hydrophila YJ-1 in $0.1 \mathrm{ml}$ of PBS. The fish were observed daily for 2 weeks after the challenge. Fish were observed to determine survival rate, and formation of ulcers and hemorrhagic lesions on the skin. Observation was made for a period of 2 weeks and data on mortality were calculated according to Amend ${ }^{38}$. Bacterial isolation was performed by cultivation from hemorrhagic and ulcerative lesions, and from visceral organs of dead fish. This was also done in all surviving fish. Animal experiments were carried out in compliance with the National Institutes of Health Guide for the Care and Use of Laboratory Animals and approved by IACUC (Institutional Animal Care and Use Committee of China Pharmaceutical University).

Data analysis. Data are presented as mean \pm SD of the number of fish per group. Data were statistically processed for one-way analysis of variance (ANOVA) to find out any significant differences among the experimental groups and the comparison between two treatments were done. Significant differences were determined at $\mathrm{P}<$ 0.05 .

1. Goossens, H. et al. Outpatient antibiotic use in Europe and association with resistance: a cross-national database study. Lancet 365 (9459), 579-587 (2005).

2. Suga, H. \& Smith, K. M. Molecular mechanisms of bacterial quorum sensing as a new drug target. Curr Opin Chem Biol. 7, 586-591 (2003).

3. Defoirdt, T., Boon, N., Bossier, P. \& Verstraete, W. Disruption of bacterial quorum sensing: an unexplored strategy to fight infections in aquaculture. Aquaculture 240, 69-88 (2004).

4. Merritt, J., Qi, F., Goodman, S. D., Anderson, M. H. \& Shi, W. Mutation of luxS affects biofilm formation in Streptococcus mutants. Infect. Immun. 71, 1972-1979 (2003).

5. Parsek, M. R. \& Greenberg, E. P. Sociomicrobiology: The connections between quorum sensing and biofilms. Trends Microbiol. 13, 27-33 (2005).

6. Cvitkovitch, D. G., Li, Y. H. \& Ellen, R. P. Quorum sensing and biofilm formation in Streptococcal infections. J. Clin. Invest. 112, 1626-1632 (2003).

7. Shih, P. C. \& Huang, C. T. Effect of quorum sensing deficiency on Pseudomonas aeruginosa biofilm formation and antibiotic resistance. J. Antimicrob. Chemoth. 49, 309-314 (2002).

8. Miller, M. B. \& Bassler, B. L. Quorum sensing in bacteria. Annu. Rev. Microbiol. 55, 165-199 (2001).

9. Waters, C. M. \& Bassler, B. L. Quorum sensing: Cell-to-cell communication in bacteria. Annu. Rev. Cell Dev. Biol. 21, 319-346 (2005).

10. Von Bodman, S. B., Willey, J. M. \& Diggle, S. P. Cell-cell communication in bacteria: United we stand. J. Bacteriol. 190, 4377-4391 (2008).

11. Federle, M. J. \& Bassler, B. L. Interspecies communication in bacteria. J. Clin. Invest. 112, 1291-1299 (2003).

12. Mellbye, B. \& Schuster, M. The sociomicrobiology of anti-virulence drug resistance: A proof of concept. MBio 2, doi:10.1128/mBio.00131-11 (2011)

13. Shrout, J. D. et al. The impact of quorum sensing and swarming motility on Pseudomonas aeruginosa biofilm formation is nutritionally conditional. Mol. Microbiol. 62, 1264-1277 (2006).
14. Tremblay, J., Richardson, A. P., Lépine, F. \& Déziel, E. Self-produced extracellular stimuli modulate the Pseudomonas aeruginosa swarming motility behaviour. Environ. Microbiol. 9, 2622-2630 (2007).

15. Finch, R. G., Pritchard, D. I., Bycroft, B. W., Williams, P. \& Stewart, G. S. A. B. Quorum sensing: a novel target for anti-infective therapy. J Antimicrob Chemother 42, 569-571 (1998).

16. Hentzer, M. et al. Attenuation of Pseudomonas aeruginosa virulence by quorum sensing inhibitors. EMBO J 22, 3803-3815 (2003).

17. Defoirdt, T., Boon, N., Bossier, P. \& Verstraete, W. Disruption of bacterial quorum sensing: an unexplored strategy to fight infections in aquaculture. Aquaculture 240, 69-88 (2004).

18. Boyen, F. et al. Quorum sensing in veterinary pathogens: Mechanisms, clinical importance and future perspectives. Veterinary Microbiology 135, 187-195 (2009).

19. Givskov, M. et al. Eukaryotic interference with homoserine lactone-mediated prokaryotic signalling. J. Bacteriol. 178, 6618-6622 (1996).

20. Roy, V., Adams, B. L. \& Bentley, W. E. Developing next generation antimicrobials by intercepting AI-2 mediated quorum sensing. Enzyme Microb. Technol. 49, 113-123 (2011)

21. Chan, K. G., Yin, W. F., Sam, C. K. \& Koh, C. L. A novel medium for the isolation of N-acylhomoserine lactone-degrading bacteria. J. Ind. Microbiol. Biotechnol. 36, 247-251 (2009).

22. Tang, K. et al. Evaluation of a new high-throughput method for identifying quorum quenching bacteria. Sci Rep. Oct 14;3:2935. doi: 10.1038/srep02935 (2013).

23. Christiaen, S. E., Brackman, G., Nelis, H. J. \& Coenye, T. Isolation and identification of quorum quenching bacteria from environmental samples. J. Microbiol. Methods 87, 213-219 (2011).

24. Kalia, V. C. Quorum Sensing Inhibitors: An overview. Biotechnology Advances 31(2), 224-245 (2013).

25. Chu, W. H., Lu, F., Zhu, W. \& Kang, C. T. Isolation and characterization of new potential probiotic bacteria based on quorum-sensing system. J. Applied Microbiol.y 110(1), 202-208 (2011).

26. Chu, W. H., Yan, J., Liu, Y. W. \& Zhu, W. Role of the quorum-sensing system in biofilm formation and virulence of Aeromonas hydrophila. Afr. J. Microbiol. Res. 5, 5819-5825 (2011).

27. Mittal, K. R., Lalonde, G., Leblanc, D., Olivier, G. \& Lallier, R. Aeromonas hydrophila in rainbow trout: relation between virulence and surface characteristics. Can. J. Microbiol. 26, 1501-1503 (1980).

28. Zhang, M. C., Cao, Y. N., Yao, B., Bai, D. Q. \& Zhou, Z. G. Characteristics of quenching enzyme AiiO-AIO6 and its effect on Aeromonas hydrophila virulence factors expression. Journal of Fisheries of China. 35(11), 1720-1728 (2011).

29. Cao, Y. A. et al. Orally administered thermostable N-acyl homoserine lactonase from Bacillus sp strain AI96 attenuates Aeromonas hydrophila infection in zebrafish. Appl Environ Microbiol 78, 1899-1908 doi: 10.1128/AEM.06139-11 (2012).

30. McLean, R. J., Pierson III, L. S. \& Fuqua, C. A simple screening protocol for the identification of quorum signal antagonists. J. Microbiol. Methods 58, 351-360 (2004).

31. Das, B. K. et al. Antagonistic activity of cellular components of Pseudomonas species against Aeromonas hydrophila. Aquaculture 253, 17-24 (2006).

32. Swift, S. et al. Quorum sensing-dependent regulation and blockade of exoprotease production in Aeromonas hydrophila. Infect. Immun. 67, 5192-5199 (1999).

33. Güven, K. \& Mutlu, M. B. Properties of Bacillus cereus collected from different food sources. Turk J Biol 33, 101-108 (2009).

34. Mao, Y. et al. The Effect of an Adding Histidine on Biological Activity and Stability of Pc-pis from Pseudosciaena crocea. PLoS ONE 8(12), e83268. doi:10.1371/ journal.pone.0083268 (2013).

35. O’Toole, G. A. \& Kolter, R. Flagellar and twitching motility are necessary for Pseudomonas aeruginosa biofilm development. Mol Microbiol 30, 295-304. doi:10.1046/j.1365-2958.1998.01062.x. (1998).

36. Gopalakannan, A. \& Arul, V. Immunomodulatory effects of dietary intake of chitin, chitosan and levamisole on the immune system of Cyprinus carpio and control of Aeromonas hydrophila infection in ponds. Aquaculture 255, 179-187 (2006).

37. Reed, L. J. \& Muench, H. A simple method of estimating fifty percent endpoints. American Journal of Hygiene 27, 493-497 (1938).

38. Amend, D. F. Potency testing of fish vaccines. Dev Bio Standard 49, 447-454 (1981).

\section{Acknowledgments}

We thank Dr. Robert J.C. McLean (Texas State University, USA) for providing the quorum sensing C. violaceum monitor strain. This work was supported by the Priority Academic Program Development of Jiangsu Higher Education Institutions (PAPD).

\section{Author contributions}

W.H.C. designed the experiments. S.X.Z., W.Z. and X.Y.Z. performed the experiments S.X.Z. analyzed the data. W.H.C. wrote the main manuscript text and S.X.Z. and W.Z. prepared figures 1-7. All authors reviewed the manuscript. 


\section{Additional information}

Competing financial interests: The authors declare no competing financial interests.

How to cite this article: Chu, W., Zhou, S., Zhu, W. \& Zhuang, X. Quorum quenching bacteria Bacillus sp. QSI-1 protect zebrafish (Danio rerio) from Aeromonas hydrophila infection. Sci. Rep. 4, 5446; DOI:10.1038/srep05446 (2014). cc) (i) $\Theta($ This work is licensed under a Creative Commons Attribution-NonCommercialNoDerivs 4.0 International License. The images or other third party material in this article are included in the article's Creative Commons license, unless indicated otherwise in the credit line; if the material is not included under the Creative Commons license, users will need to obtain permission from the license holder in order to reproduce the material. To view a copy of this license, visit http:// creativecommons.org/licenses/by-nc-nd/4.0/ 